\title{
Change in blood test results prior to diagnosis in multiple myeloma
}

\author{
Authors: Catherine Atkin, ${ }^{\mathrm{A}}$ Elizabeth Sapey ${ }^{\mathrm{A}}$ and Alex Richter ${ }^{\mathrm{A}}$
}

\section{Introduction}

Multiple myeloma is a plasma cell malignancy, with one of the longest diagnostic pathways of all cancers in the UK. ${ }^{1}$ Delay in diagnosis in myeloma is associated with poorer prognosis and more advanced disease. ${ }^{2}$ End organ damage is often present at diagnosis, ${ }^{3}$ detectable on blood tests as anaemia, renal impairment and hypercalcaemia - recognition often relies on these features. Other blood test abnormalities occur, including raised total protein, or raised calculated globulin (total protein minus albumin) which reflects total immunoglobulin levels. ${ }^{4}$ Previous research suggests that some abnormalities may be present for years before diagnosis. ${ }^{5,6}$ This may allow strategies to detect these changes earlier, and prompt automated testing to reduce diagnostic delay. This research aimed to assess blood test abnormalities present at diagnosis with multiple myeloma, and change over time before diagnosis.

\section{Methods}

Data were collected for all patients diagnosed with multiple myeloma at the Queen Elizabeth Hospital Birmingham between 2007 and 2017. Patients were identified using clinical coding. Blood test results available on clinical systems up to the date of diagnosis were collated. Calculated globulin was determined for each result and compared with immunoglobulin results from the same sample using Spearman's rank order correlation. Test results were grouped in 90-day intervals preceding date of diagnosis. Mean results for each test were calculated for each interval and change was assessed using one-way repeated measures ANOVA.

\section{Results}

Using the above criteria, 285 patients were identified. In total, 56,711 individual tests were available. Per patient, this ranged from 10 results (one full blood count) to 2,535 individual tests spanning 13 years. The most common abnormalities at diagnosis were low haematocrit (86\%), anaemia (81\%), raised total protein $(49.8 \%)$ and raised creatinine $(44.5 \%)$.

There was a statistically significant decline in mean haemoglobin over 2 years prior to diagnosis $(\mathrm{p}<0.0005)$.

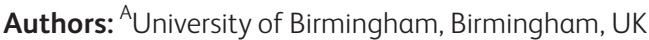

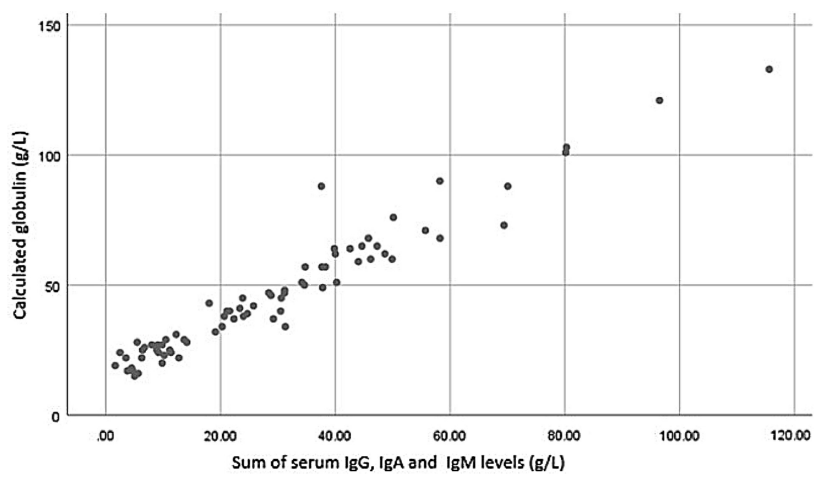

Fig 1. Correlation between calculated globulin and sum total of immunoglobulin G, immunoglobulin A and immunoglobulin M.

Haematocrit appeared to decline over the same time, but this was not statistically significant. There appeared to be an increase in mean corrected calcium and creatinine in the 90 days preceding diagnosis, but not before.

Calculated globulin ranged from $13 \mathrm{~g} / \mathrm{L}$ to $133 \mathrm{~g} / \mathrm{L}$, and was raised in $43.8 \%$ of patients and low in $2.5 \%$. Calculated globulin correlated well with total immunoglobulin levels (combined $\operatorname{IgG}$, IgA and IgM) with a strong positive correlation (rs (73) 0.961; p<0.0005; Fig 1). However, the relationship between calculated globulin and each immunoglobulin class separately was not linear (Fig 2).

\section{Discussion}

Abnormalities that are often used to prompt testing, for instance hypercalcaemia, raised total protein and abnormal renal function, are abnormal in fewer than half of patients with myeloma at diagnosis. It is important to highlight this in education and awareness campaigns, as normal results should not prevent testing.

Haemoglobin declines over at least 2 years prior to diagnosis. A gradual unexplained fall in haemoglobin could be used to identify patients for testing.

Calculated globulin is an accurate reflection of the total immunoglobulin level. An abnormal calculated globulin may prompt testing for myeloma, but normal calculated globulin cannot rule out myeloma, due to a fall in the uninvolved classes of immunoglobulin, so cannot be used alone as a screening test. 
a)

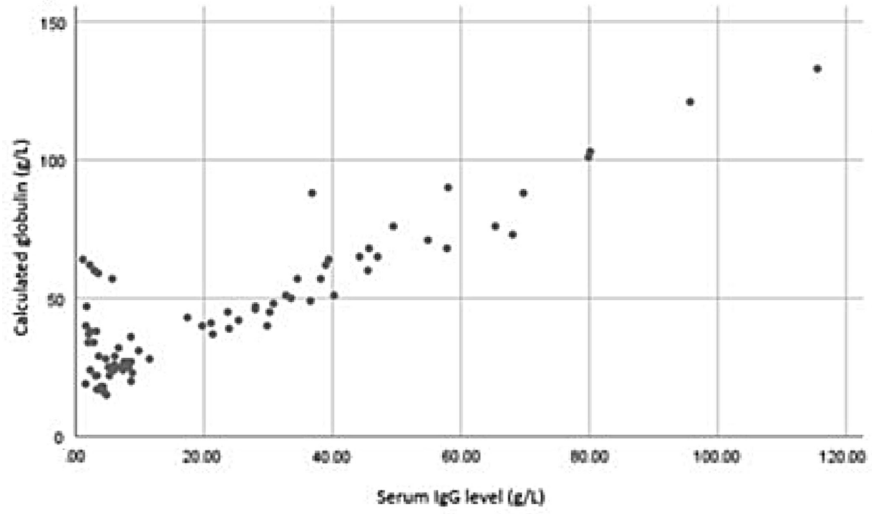

b)

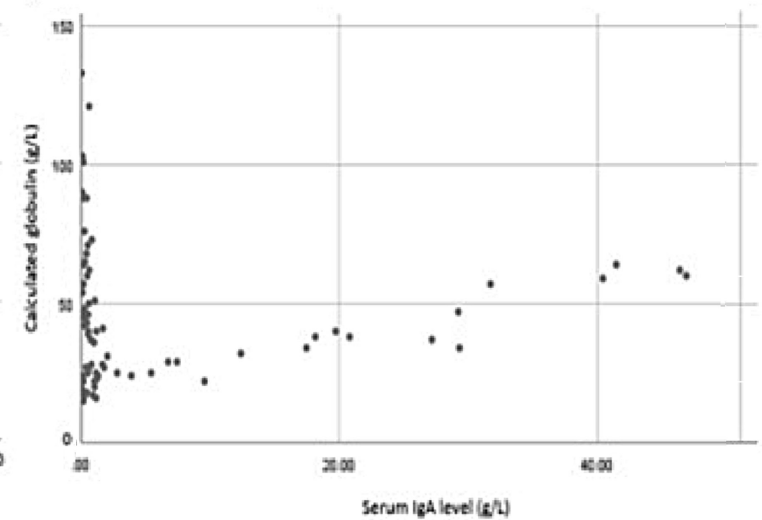

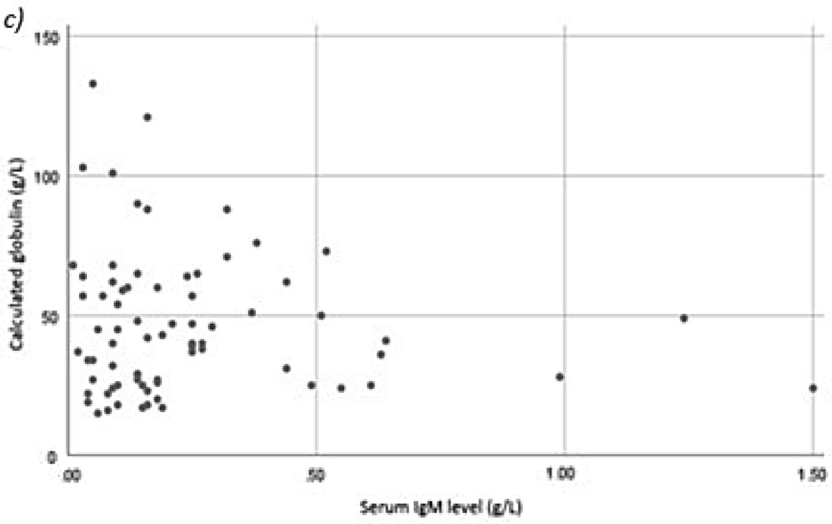

Fig 2. Correlation between calculated globulin and individual classes of immunoglobulin.

\section{Conflicts of interest}

None declared.

\section{References}

1 Howell D, Smith A, Jack A et al. Time-to-diagnosis and symptoms of myeloma, lymphomas and leukaemias: a report from the Haematological Malignancy Research Network. BMC Hematol 2013;13:9.

2 Kariyawasan CC, Hughes DA, Jayatillake MM, Mehta AB. Multiple myeloma: causes and consequences of delay in diagnosis. QJM 2007;100:635-40.
3 Rajkumar SV, Dimpoulos MA, Palumbo A et al. International Myeloma Working Group updated criteria for the diagnosis of multiple myeloma. Lancet Oncol 2014;15:e538-48.

4 Jolles S, Borrell R, Zouwail S et al. Calculated globulin (CG) as a screening test for antibody deficiency. Clin Exp Immunol 2014;177:671-8.

5 Edgren G, Bagnardi V, Bellocco R et al. Pattern of declining hemoglobin concentration before cancer diagnosis. Int $\mathrm{J}$ Cancer 2010;127:1429-36.

6 Koshiaris C, Van den Bruel A, Oke JL et al. Early detection of multiple myeloma in primary care using blood tests: a case-control study in primary care. Br J Gen Pract 2018;68:e586-93. 